\title{
SMALL $\mathrm{ScC}_{\mathrm{n}}$ CYCLIC CLUSTERS. A DENSITY FUNCTIONAL STUDY OF THEIR STRUCTURE AND STABILITY.
}

\author{
Supporting Information \\ Pilar Redondo, Carmen Barrientos, and Antonio Largo* \\ Departamento de Química Física \\ Facultad de Ciencias \\ Universidad de Valladolid \\ 47005 Valladolid \\ Spain \\ * Author to whom correspondence should be addressed. \\ e-mail: alargo@qf.uva.es
}

Fax: 34-983-423013 
Table S1: Vibrational frequencies and rotational constants for cyclic $\mathrm{ScC}_{\mathrm{n}}$ clusters with the B3LYP/6-311+G(d) (first line) and B3LYP/LAN-6+(d) (second line) methods.

\begin{tabular}{|c|c|c|c|}
\hline Isomer & State & Vibrational frequencies $\left(\mathrm{cm}^{-1}\right)$ & $\begin{array}{c}\text { Rotational constants } \\
(\mathrm{GHz})\end{array}$ \\
\hline $\mathrm{ScC}_{2}$ & ${ }^{2} \mathrm{~A}_{1}$ & $\begin{array}{l}329 / 635 / 1828 \\
322 / 629 / 1830 \\
280 / 392 / 1711 \\
235 / 403 / 1708 \\
\end{array}$ & $\begin{array}{l}52.649 / 8.384 / 7.233 \\
52.687 / 8.407 / 7.250 \\
51.445 / 6.951 / 6.123 \\
51.395 / 7.041 / 6.193 \\
\end{array}$ \\
\hline $\mathrm{ScC}_{3}$ & ${ }^{2} \mathrm{~A}_{2}$ & $\begin{array}{l}374 / 418 / 591 / 772 / 1252 / 1524 \\
375 / 411 / 589 / 770 / 1256 / 1539 \\
356 / 447 / 521 / 631 / 1273 / 1515 \\
353 / 444 / 519 / 622 / 1277 / 1519\end{array}$ & $\begin{array}{l}13.444 / 7.780 / 4.928 \\
13.461 / 7.771 / 4.927 \\
13.674 / 6.777 / 4.531 \\
13.782 / 6.793 / 4.543\end{array}$ \\
\hline $\mathrm{ScC}_{4}$ & ${ }^{2} \mathrm{~A}_{1}$ & $\begin{array}{l}274 / 373 / 448 / 466 / 481 / 625 / 1051 / 1859 / 1982 \\
273 / 374 / 437 / 465 / 476 / 609 / 1054 / 1854 / 1972 \\
261 / 306 / 360 / 433 / 500 / 524 / 1016 / 1479 / 1904 \\
250 / 294 / 589 / 426 / 500 / 510 / 1015 / 1492 / 1905\end{array}$ & $\begin{array}{l}6.329 / 6.307 / 3.159 \\
6.338 / 6.335 / 3.168 \\
6.187 / 5.626 / 2.947 \\
6.192 / 5.614 / 2.944\end{array}$ \\
\hline $\mathrm{ScC}_{5}$ & ${ }^{2} \mathrm{~A}^{\prime}$ & $\begin{array}{l}131 / 364 / 380 / 399 / 455 / 489 / 639 / 639 / 976 / 1386 / 1700 / 1724 \\
136 / 356 / 376 / 396 / 452 / 497 / 631 / 633 / 979 / 1384 / 1704 / 1727 \\
103 / 297 / 327 / 368 / 386 / 418 / 508 / 587 / 965 / 1430 / 1605 / 1746 \\
104 / 293 / 319 / 367 / 381 / 406 / 496 / 572 / 965 / 1429 / 1607 / 1748\end{array}$ & $\begin{array}{l}6.608 / 3.676 / 2.439 \\
6.566 / 3.698 / 2.456 \\
5.725 / 3.581 / 2.203 \\
5.723 / 3.583 / 2.203\end{array}$ \\
\hline $\mathrm{ScC}_{6}$ & ${ }^{2} \mathrm{~A}_{1}$ & $\begin{array}{l}134 / 233 / 285 / 299 / 330 / 405 / 420 / 456 / 533 / 596 / 881 / 1221 / 1880 / 1984 / 2086 \\
138 / 233 / 274 / 293 / 327 / 395 / 418 / 447 / 512 / 555 / 882 / 1221 / 1879 / 1984 / 2081 \\
71 / 164 / 218 / 229 / 322 / 328 / 338 / 521 / 526 / 556 / 844 / 1197 / 1545 / 1750 / 1918 \\
85 / 172 / 204 / 209 / 319 / 329 / 346 / 509 / 520 / 546 / 844 / 1194 / 1556 / 1757 / 1920\end{array}$ & $\begin{array}{l}5.123 / 2.380 / 1.625 \\
5.134 / 2.379 / 1.626 \\
5.546 / 2.210 / 1.580 \\
5.601 / 2.187 / 1.573\end{array}$ \\
\hline $\mathrm{ScC}_{7}$ & $\begin{array}{l}{ }^{2} \mathrm{~A} " \\
{ }^{2} \mathrm{~A}_{2} \\
{ }^{4} \mathrm{~B}_{1}\end{array}$ & $\begin{array}{l}33 / 60 / 305 / 305 / 332 / 357 / 358 / 389 / 418 / 543 / 560 / 617 / 881 / 1097 / 1500 / 1689 / \\
1801 / 1836 \\
60 \mathrm{i} / 76 / 282 / 284 / 348 / 352 / 353 / 386 / 387 / 525 / 556 / 610 / 876 / 1100 / 1506 / 1695 / \\
1802 / 1837 \\
102 / 157 / 207 / 247 / 287 / 316 / 328 / 355 / 441 / 448 / 480 / 591 / 842 / 1121 / 1539 / \\
1577 / 1826 / 1844 \\
109 / 160 / 195 / 236 / 281 / 298 / 329 / 354 / 437 / 440 / 466 / 593 / 842 / 1121 / 1533 / \\
1582 / 1828 / 1844\end{array}$ & $\begin{array}{l}4.281 / 1.949 / 1.368 \\
4.491 / 1.902 / 1.336 \\
3.986 / 1.810 / 1.245 \\
3.981 / 1.812 / 1.245\end{array}$ \\
\hline $\mathrm{ScC}_{8}$ & ${ }^{2} \mathrm{~A}_{1}$ & $\begin{array}{l}48 / 67 / 140 / 143 / 176 / 261 / 279 / 373 / 384 / 426 / 471 / 501 / 530 / 531 / 824 / 985 / \\
1306 / 1922 / 1942 / 2017 / 2106 \\
68 / 92 / 95 / 147 / 249 / 262 / 295 / 384 / 396 / 435 / 451 / 498 / 532 / 532 / 841 / 1022 / \\
1324 / 1966 / 1993 / 2054 / 2131 \\
27 / 132 / 239 / 241 / 258 / 316 / 356 / 364 / 446 / 470 / 489 / 505 / 604 / 632 / 822 / 1016 / \\
1256 / 1358 / 1682 / 1735 / 1930 \\
86 / 100 / 165 / 237 / 256 / 326 / 356 / 375 / 397 / 447 / 463 / 511 / 583 / 635 / 816 / 1036 / \\
1223 / 1408 / 1684 / 1750 / 1961\end{array}$ & $\begin{array}{l}1.832 / 1.705 / 0.883 \\
1.857 / 1.450 / 0.814 \\
3.257 / 1.548 / 1.104 \\
3.096 / 1.584 / 1.087\end{array}$ \\
\hline $\mathrm{ScC}_{9}$ & ${ }^{4} \mathrm{~B}_{1}$ & $\begin{array}{l}70 / 114 / 130 / 192 / 201 / 214 / 372 / 389 / 421 / 433 / 456 / 468 / 485 / 567 / 575 / 589 / \\
792 / 855 / 1278 / 1328 / 1800 / 1880 / 1897 / 2020 \\
55 / 111 / 115 / 143 / 181 / 207 / 372 / 395 / 428 / 436 / 451 / 469 / 474 / 552 / 552 / 592 / \\
789 / 861 / 1285 / 1343 / 1798 / 1880 / 1896 / 2020 \\
60 / 71 / 74 / 134 / 145 / 193 / 345 / 365 / 380 / 401 / 411 / 452 / 491 / 493 / 523 / 545 / 803 / \\
939 / 1249 / 1409 / 1679 / 1923 / 1942 / 1956 \\
66 / 71 / 82 / 137 / 145 / 195 / 343 / 367 / 373 / 399 / 415 / 459 / 485 / 496 / 507 / 538 / 807 / \\
942 / 1250 / 1407 / 1678 / 1921 / 1943 / 1958\end{array}$ & $\begin{array}{l}1.728 / 1.556 / 0.819 \\
1.705 / 1.560 / 0.815 \\
1.566 / 0.968 / 0.598 \\
1.585 / 0.951 / 0.594\end{array}$ \\
\hline $\mathrm{ScC}_{10}$ & $\begin{array}{l}{ }^{2} \mathrm{~B}_{2} \\
{ }^{2} \mathrm{~A}^{\prime} \\
{ }^{4} \mathrm{~A}^{\prime}\end{array}$ & $\begin{array}{l}28 / 54 / 65 / 94 / 153 / 188 / 194 / 285 / 318 / 367 / 412 / 417 / 422 / 433 / 513 / 518 / 531 / 55 \\
7 / 738 / 835 / 1214 / 1426 / 1812 / 1878 / 1901 / 2024 / 2086 \\
48 / 98 / 99 / 150 / 187 / 267 / 290 / 309 / 350 / 378 / 396 / 407 / 427 / 451 / 490 / 508 / 530 / 5 \\
70 / 727 / 929 / 1179 / 1380 / 1785 / 1876 / 1946 / 1991 / 2072 \\
36 / 107 / 108 / 118 / 158 / 249 / 273 / 289 / 380 / 387 / 397 / 435 / 456 / 460 / 504 / 504 / 54 \\
6 / 607 / 721 / 886 / 1128 / 1405 / 1550 / 1716 / 1794 / 1884 / 1981 \\
44 / 108 / 112 / 123 / 164 / 256 / 288 / 306 / 378 / 398 / 413 / 449 / 462 / 480 / 507 / 527 / 54 \\
0 / 603 / 726 / 887 / 11311400 / 1550 / 1722 / 1795 / 1887 / 1982 \\
\end{array}$ & $\begin{array}{l}1.309 / 1.211 / 0.629 \\
1.795 / 0.939 / 0.616 \\
1.597 / 0.866 / 0.562 \\
1.611 / 0.863 / 0.562\end{array}$ \\
\hline
\end{tabular}


Table S2: Vibrational frequencies and rotational constants for cyclic $\mathrm{ScC}_{\mathrm{n}}{ }^{+}$clusters with the B3LYP/6-311+G(d) (first line) and B3LYP/LAN-6+(d) (second line) methods.

\begin{tabular}{|c|c|c|c|}
\hline Isomer & State & Vibrational frequencies $\left(\mathrm{cm}^{-1}\right)$ & $\begin{array}{l}\text { Rotational constants } \\
(\mathrm{GHz})\end{array}$ \\
\hline $\mathrm{ScC}_{2}^{+}$ & ${ }^{3} \mathrm{~A}_{2}$ & $\begin{array}{l}451 / 734 / 1803 \\
451 / 745 / 1807 \\
259 / 495 / 1840 \\
230 / 499 / 1845\end{array}$ & $\begin{array}{l}52.270 / 9.153 / 7.789 \\
52.321 / 9.209 / 7.830 \\
52.863 / 7.100 / 6.259 \\
52.948 / 7.150 / 6.299\end{array}$ \\
\hline $\mathrm{ScC}_{3}^{+}$ & ${ }^{1} \mathrm{~A}_{1}$ & $\begin{array}{l}248 / 473 / 625 / 937 / 1035 / 1412 \\
257 / 479 / 631 / 940 / 1033 / 1418 \\
397 / 454 / 548 / 662 / 1312 / 1564 \\
397 / 448 / 538 / 651 / 1318 / 1580\end{array}$ & $\begin{array}{l}41.514 / 4.370 / 3.954 \\
41.585 / 4.378 / 3.961 \\
14.137 / 7.137 / 4.742 \\
14.211 / 7.118 / 4.743\end{array}$ \\
\hline $\mathrm{ScC}_{4}^{+}$ & ${ }^{3} \mathrm{~A}_{1}$ & $\begin{array}{l}330 / 442 / 506 / 508 / 572 / 648 / 1106 / 1815 / 1941 \\
337 / 442 / 501 / 504 / 578 / 634 / 1112 / 1809 / 1936 \\
164 / 374 / 430 / 531 / 537 / 564 / 1079 / 1548 / 1742 \\
163 / 371 / 423 / 525 / 530 / 550 / 1081 / 1564 / 1785\end{array}$ & $\begin{array}{l}6.750 / 6.647 / 3.349 \\
6.746 / 6.697 / 3.358 \\
6.659 / 6.221 / 3.216 \\
6.640 / 6.243 / 3.218\end{array}$ \\
\hline $\mathrm{ScC}_{5}^{+}$ & ${ }^{1} \mathrm{~A}_{1}$ & $\begin{array}{l}108 / 300 / 341 / 358 / 453 / 553 / 586 / 595 / 982 / 1414 / 1785 / 1819 \\
113 / 297 / 327 / 349 / 444 / 542 / 572 / 583 / 983 / 1412 / 1788 / 1827 \\
160 / 318 / 376 / 405 / 486 / 517 / 535 / 638 / 1008 / 1406 / 1644 / 1743 \\
167 / 300 / 371 / 404 / 484 / 514 / 523 / 624 / 1012 / 1397 / 1655 / 1748\end{array}$ & $\begin{array}{l}6.714 / 3.494 / 2.298 \\
6.743 / 3.497 / 2.303 \\
5.677 / 3.950 / 2.403 \\
5.606 / 3.997 / 2.414\end{array}$ \\
\hline $\mathrm{ScC}_{6}^{+}$ & $\begin{array}{l}{ }^{1} \mathrm{~A}_{1} \\
{ }^{1} \mathrm{~A}, \\
{ }^{3} \mathrm{~A},\end{array}$ & $\begin{array}{l}50 / 242 / 358 / 359 / 402 / 429 / 448 / 484 / 565 / 628 / 921 / 1244 / 1844 / 1946 / 2049 \\
9 / 240 / 335 / 353 / 397 / 426 / 436 / 468 / 538 / 566 / 923 / 1246 / 1839 / 1940 / 2041 \\
121 / 210 / 285 / 321 / 346 / 383 / 428 / 460 / 507 / 538 / 953 / 1254 / 1596 / 1729 / 1838 \\
124 / 207 / 284 / 305 / 327 / 358 / 424 / 426 / 482 / 507 / 958 / 1258 / 1610 / 1744 / 1840\end{array}$ & $\begin{array}{l}5.445 / 2.548 / 1.736 \\
5.476 / 2.555 / 1.744 \\
4.955 / 2.548 / 1.817 \\
4.901 / 2.560 / 1.829\end{array}$ \\
\hline $\mathrm{ScC}_{7}^{+}$ & ${ }^{3} \mathrm{~B}_{1}$ & $\begin{array}{l}123 / 137 / 160 / 224 / 314 / 360 / 425 / 434 / 518 / 520 / 566 / 633 / 992 / 1136 / 1463 / \\
1718 / 1902 / 1920 \\
125 / 136 / 170 / 230 / 309 / 367 / 421 / 430 / 510 / 511 / 562 / 627 / 1006 / 1156 / 1487 / \\
1711 / 1891 / 1916 \\
144 / 154 / 158 / 207 / 366 / 378 / 412 / 466 / 469 / 501 / 545 / 586 / 977 / 1153 / 1540 / \\
1564 / 1855 / 1889 \\
142 / 158 / 159 / 214 / 367 / 386 / 410 / 468 / 482 / 532 / 544 / 588 / 985 / 1163 / 1541 / \\
1568 / 1853 / 1886\end{array}$ & $\begin{array}{l}2.944 / 1.663 / 1.075 \\
3.017 / 1.622 / 1.067 \\
2.894 / 1.634 / 1.044 \\
2.944 / 1.606 / 1.039\end{array}$ \\
\hline $\mathrm{ScC}_{8}^{+}$ & ${ }^{3} \mathrm{~A} "$ & $\begin{array}{l}57 / 140 / 156 / 161 / 280 / 314 / 328 / 387 / 426 / 468 / 474 / 518 / 582 / 602 / 896 / 1064 / \\
1366 / 1931 / 1966 / 2029 / 2097 \\
49 / 143 / 161 / 163 / 281 / 314 / 335 / 410 / 424 / 474 / 481 / 532 / 586 / 604 / 900 / 1070 / \\
1371 / 1926 / 1958 / 2025 / 2094 \\
86 / 106 / 127 / 182 / 246 / 256 / 283 / 333 / 354 / 381 / 421 / 428 / 457 / 479 / 906 / 1094 / \\
1419 / 1884 / 1912 / 1957 / 2010 \\
106 / 112 / 127 / 187 / 245 / 282 / 289 / 354 / 358 / 386 / 438 / 439 / 458 / 482 / 910 / 1097 / 1 \\
421 / 1888 / 1918 / 1958 / 2013\end{array}$ & $\begin{array}{l}2.059 / 1.343 / 0.813 \\
2.079 / 1.332 / 0.812 \\
2.076 / 1.146 / 0.738 \\
\\
2.098 / 1.136 / 0.737\end{array}$ \\
\hline $\mathrm{ScC}_{9}^{+}$ & ${ }^{3} \mathrm{~B}_{1}$ & $\begin{array}{l}\text { 94/101/123/145/181/257/336/373/403/421/455/520/533/539/556/599/ } \\
874 / 992 / 1281 / 1487 / 1742 / 1945 / 1955 / 1979 \\
92 / 102 / 127 / 153 / 188 / 260 / 348 / 381 / 399 / 418 / 450 / 523 / 525 / 528 / 560 / 590 / \\
878 / 1004 / 1287 / 1500 / 1737 / 1925 / 1954 / 1980 \\
87 / 91 / 128 / 147 / 187 / 224 / 361 / 380 / 400 / 408 / 436 / 474 / 483 / 485 / 523 / 533 / 849 / \\
999 / 1286 / 1407 / 1641 / 1893 / 1916 / 1940 \\
95 / 95 / 130 / 154 / 194 / 239 / 356 / 382 / 389 / 402 / 435 / 444 / 488 / 500 / 510 / 513 / 854 / \\
1011 / 1294 / 1404 / 1647 / 1895 / 1919 / 1943\end{array}$ & $\begin{array}{l}1.751 / 0.912 / 0.600 \\
1.770 / 0.901 / 0.597 \\
1.747 / 0.900 / 0.594 \\
1.775 / 0.883 / 0.590\end{array}$ \\
\hline $\mathrm{ScC}_{10}^{+}$ & ${ }^{3} \mathrm{~B}_{2}$ & $\begin{array}{l}85 / 116 / 124 / 140 / 212 / 218 / 232 / 317 / 330 / 369 / 415 / 432 / 437 / 449 / 499 / 503 / \\
513 / 526 / 825 / 967 / 1249 / 1458 / 1877 / 1978 / 1986 / 2113 / 2118 \\
92 / 118 / 131 / 148 / 216 / 233 / 250 / 325 / 339 / 406 / 413 / 444 / 448 / 454 / 517 / 519 / \\
568 / 596 / 833 / 975 / 1257 / 1467 / 1853 / 1974 / 1979 / 2109 / 2114 \\
68 / 109 / 114 / 142 / 167 / 224 / 226 / 308 / 325 / 337 / 350 / 375 / 398 / 425 / 456 / 471 / \\
491 / 495 / 807 / 964 / 1248 / 1527 / 1693 / 1813 / 1863 / 1972 / 2011 \\
75 / 110 / 121 / 148 / 188 / 235 / 239 / 315 / 340 / 347 / 378 / 390 / 395 / 430 / 509 / 511 / \\
523 / 525 / 810 / 969 / 1250 / 1531 / 1674 / 1820 / 1864 / 1973 / 2014\end{array}$ & $\begin{array}{l}1.519 / 0.660 / 0.460 \\
1.548 / 0.654 / 0.460 \\
1.405 / 0.673 / 0.455 \\
1.425 / 0.666 / 0.454\end{array}$ \\
\hline
\end{tabular}


Table S3: Vibrational frequencies and rotational constants for cyclic $\mathrm{ScC}_{\mathrm{n}}{ }^{-}$clusters with the B3LYP/6-311+G(d) (first line) and B3LYP/LAN-6+(d) (second line) methods.

\begin{tabular}{|c|c|c|c|}
\hline Isomer & State & Vibrational frequencies $\left(\mathrm{cm}^{-1}\right)$ & $\begin{array}{l}\text { Rotational constants } \\
\qquad(\mathrm{GHz})\end{array}$ \\
\hline $\mathrm{ScC}_{2}^{-}$ & ${ }^{1} \mathrm{~A}^{\prime}$ & $\begin{array}{l}166 / 660 / 1812 \\
156 / 709 / 1806 \\
319 / 500 / 1826 \\
301 / 505 / 1829\end{array}$ & $\begin{array}{l}61.365 / 7.072 / 6.341 \\
67.876 / 6.732 / 6.125 \\
52.604 / 7.414 / 6.498 \\
52.646 / 7.451 / 6.528\end{array}$ \\
\hline $\mathrm{ScC}_{3}^{-}$ & ${ }^{1} \mathrm{~A}_{1}$ & $\begin{array}{l}260 / 351 / 517 / 977 / 1078 / 1470 \\
267 / 349 / 527 / 974 / 1080 / 1472 \\
331 / 430 / 538 / 755 / 1223 / 1533 \\
330 / 425 / 537 / 751 / 1226 / 1544\end{array}$ & $\begin{array}{l}41.211 / 3.975 / 3.625 \\
41.280 / 4.005 / 3.651 \\
13.151 / 7.302 / 4.695 \\
13.178 / 7.314 / 4.703\end{array}$ \\
\hline $\mathrm{ScC}_{4}^{-}$ & ${ }^{1} \mathrm{~A}_{1}$ & $\begin{array}{l}238 / 255 / 320 / 384 / 510 / 590 / 992 / 1778 / 1889 \\
222 / 253 / 306 / 417 / 510 / 562 / 987 / 1759 / 1888 \\
233 / 275 / 333 / 519 / 552 / 565 / 997 / 1684 / 1871 \\
234 / 269 / 325 / 514 / 550 / 555 / 996 / 1693 / 1871\end{array}$ & $\begin{array}{l}6.251 / 5.596 / 2.953 \\
6.408 / 5.538 / 2.971 \\
6.783 / 5.709 / 3.100 \\
6.793 / 5.707 / 3.102\end{array}$ \\
\hline $\mathrm{ScC}_{5}^{-}$ & $\begin{array}{l}{ }^{1} A^{\prime} \\
{ }^{3} B_{1}\end{array}$ & $\begin{array}{l}217 / 343 / 391 / 465 / 470 / 584 / 671 / 747 / 958 / 1227 / 1725 / 1741 \\
219 / 341 / 388 / 463 / 471 / 585 / 668 / 742 / 961 / 1225 / 1726 / 1744 \\
70 / 287 / 304 / 389 / 390 / 408 / 624 / 629 / 931 / 1378 / 1701 / 1754 \\
88 / 272 / 302 / 390 / 388 / 405 / 621 / 626 / 931 / 1379 / 1703 / 1753\end{array}$ & $\begin{array}{l}6.244 / 3.798 / 2.597 \\
6.238 / 3.805 / 2.605 \\
6.517 / 3.407 / 2.237 \\
6.581 / 3.396 / 2.240\end{array}$ \\
\hline $\mathrm{ScC}_{6}^{-}$ & ${ }^{1} \mathrm{~A}_{1}$ & $\begin{array}{l}67 / 154 / 243 / 264 / 400 / 402 / 469 / 504 / 578 / 618 / 861 / 1104 / 1589 / 1735 / 1843 \\
63 / 111 / 242 / 249 / 388 / 395 / 475 / 484 / 574 / 609 / 860 / 1102 / 1597 / 1740 / 1849 \\
87 / 221 / 230 / 230 / 394 / 413 / 428 / 475 / 522 / 550 / 837 / 1147 / 1711 / 1838 / 1926 \\
100 / 211 / 212 / 229 / 399 / 412 / 425 / 462 / 517 / 543 / 836 / 1145 / 1720 / 1842 / 1927\end{array}$ & $\begin{array}{l}6416 / 2.256 / 1.669 \\
6.480 / 2.236 / 1.662 \\
5.899 / 2.193 / 1.599 \\
5.940 / 2.175 / 1.592\end{array}$ \\
\hline $\mathrm{ScC}_{7}^{-}$ & ${ }^{3} \mathrm{~A}_{2}$ & $\begin{array}{l}79 / 213 / 262 / 336 / 379 / 388 / 413 / 443 / 533 / 579 / 604 / 681 / 869 / 1031 / 1322 / \\
1796 / 1818 / 1903 \\
87 / 213 / 240 / 335 / 380 / 388 / 410 / 434 / 538 / 563 / 599 / 677 / 870 / 1032 / 1312 / \\
1791 / 1822 / 1913 \\
60 / 170 / 223 / 235 / 283 / 339 / 346 / 353 / 383 / 510 / 543 / 634 / 823 / 1056 / 1484 / \\
1712 / 1837 / 1866 \\
77 / 173 / 213 / 222 / 280 / 336 / 344 / 351 / 378 / 504 / 538 / 621 / 822 / 1054 / 1482 / \\
1711 / 1837 / 1867\end{array}$ & $\begin{array}{l}4.284 / 1.93971 .413 \\
4.277 / 1.940 / 1.419 \\
4.362 / 1.752 / 1.250 \\
4.383 / 1.744 / 1.248\end{array}$ \\
\hline $\mathrm{ScC}_{8}^{-}$ & ${ }^{3} \mathrm{~A}_{1}$ & $\begin{array}{l}73 / 113 / 161 / 256 / 329 / 349 / 389 / 412 / 442 / 461 / 465 / 510 / 626 / 652 / 815 / 916 / \\
1157 / 1564 / 1814 / 1866 / 1939 \\
61 / 117 / 152 / 266 / 321 / 347 / 383 / 421 / 430 / 457 / 466 / 505 / 621 / 646 / 814 / 917 / \\
1159 / 1563 / 1813 / 1864 / 1938 \\
90 / 120 / 148 / 164 / 187 / 239 / 290 / 351 / 399 / 416 / 428 / 480 / 559 / 617 / 787 / 971 / \\
1248 / 1688 / 1889 / 1909 / 1979 \\
101 / 102 / 150 / 180 / 183 / 231 / 276 / 356 / 401 / 404 / 427 / 479 / 554 / 619 / 788 / 970 / \\
1242 / 1685 / 1889 / 1910 / 1977\end{array}$ & $\begin{array}{l}3.328 / 1.478 / 1.024 \\
3.349 / 1.473 / 1.023 \\
3129 / 1.457 / 0.994 \\
3.133 / 1.452 / 0.992\end{array}$ \\
\hline $\mathrm{ScC}_{9}^{-}$ & ${ }^{3} \mathrm{~B}_{1}$ & $\begin{array}{l}83 / 101 / 172 / 176 / 242 / 286 / 360 / 373 / 426 / 429 / 444 / 475 / 499 / 575 / 606 / 608 / \\
803 / 827 / 1294 / 1338 / 1827 / 1846 / 1881 / 2076 \\
82 / 99 / 180 / 185 / 234 / 283 / 355 / 358 / 414 / 423 / 439 / 477 / 487 / 573 / 593 / 597 / 802 / \\
822 / 1298 / 1333 / 1819 / 1844 / 1878 / 2072 \\
97 / 108 / 121 / 167 / 194 / 203 / 302 / 351 / 363 / 401 / 456 / 460 / 470 / 546 / 548 / 598 / \\
775 / 842 / 1232 / 1300 / 1816 / 1903 / 1921 / 2018 \\
97 / 103 / 118 / 144 / 179 / 196 / 306 / 352 / 363 / 400 / 451 / 451 / 456 / 519 / 524 / 594 / \\
772 / 844 / 1234 / 1306 / 1812 / 1904 / 1921 / 2018\end{array}$ & $\begin{array}{l}1.731 / 1.610 / 0.834 \\
1.717 / 1.629 / 0.836 \\
1.760 / 1.410 / 0.783 \\
1.739 / 1.415 / 0.780\end{array}$ \\
\hline $\mathrm{ScC}_{10}{ }^{-}$ & ${ }^{3} \mathrm{~B}_{2}$ & $\begin{array}{l}93 / 116 / 128 / 187 / 205 / 275 / 293 / 333 / 369 / 410 / 431 / 444 / 492 / 506 / 518 / 532 / \\
555 / 629 / 744 / 812 / 1189 / 1371 / 1639 / 1789 / 1821 / 1899 / 2086 \\
96 / 116 / 129 / 190 / 203 / 275 / 299 / 325 / 373 / 414 / 428 / 444 / 492 / 503 / 524 / 530 / \\
548 / 626 / 744 / 814 / 1191 / 1373 / 1642 / 1787 / 1820 / 1900 / 2086 \\
61 / 79 / 89 / 104 / 177 / 192 / 234 / 264 / 325 / 368 / 390 / 427 / 428 / 455 / 485 / 502 / 528 / \\
534 / 694 / 786 / 1160 / 1327 / 1734 / 1939 / 1945 / 2043 / 2080 \\
45 / 78 / 97 / 107 / 178 / 185 / 228 / 266 / 328 / 371 / 392 / 400 / 407 / 443 / 478 / 478 / 511 / \\
522 / 695 / 790 / 1160 / 1333 / 1740 / 1937 / 1843 / 2038 / 2077\end{array}$ & $\begin{array}{l}1.372 / 1.221 / 0.646 \\
1.377 / 1.221 / 0.647 \\
1.235 / 1.197 / 0.608 \\
1.235 / 1.196 / 0.607\end{array}$ \\
\hline
\end{tabular}



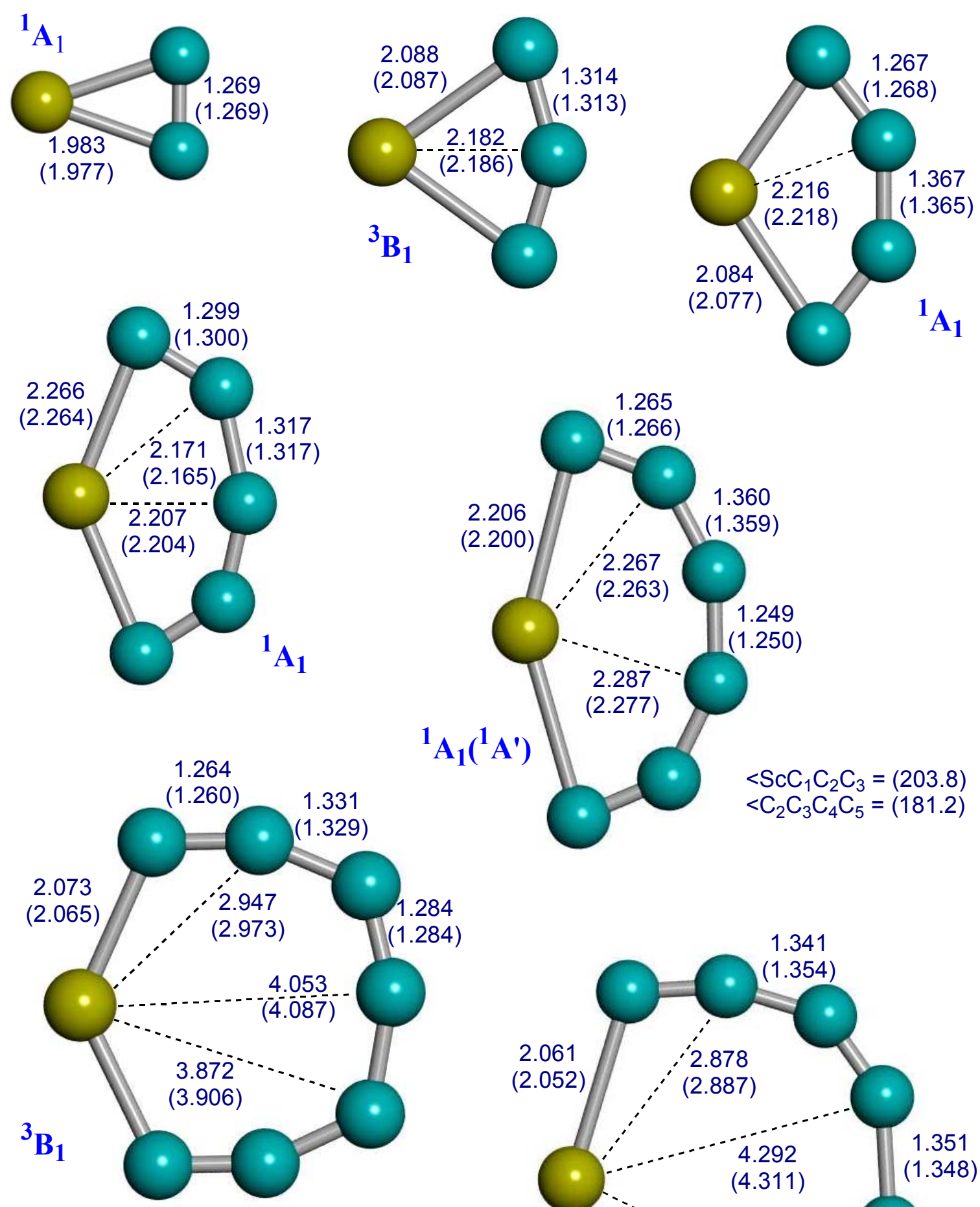

Figure S1
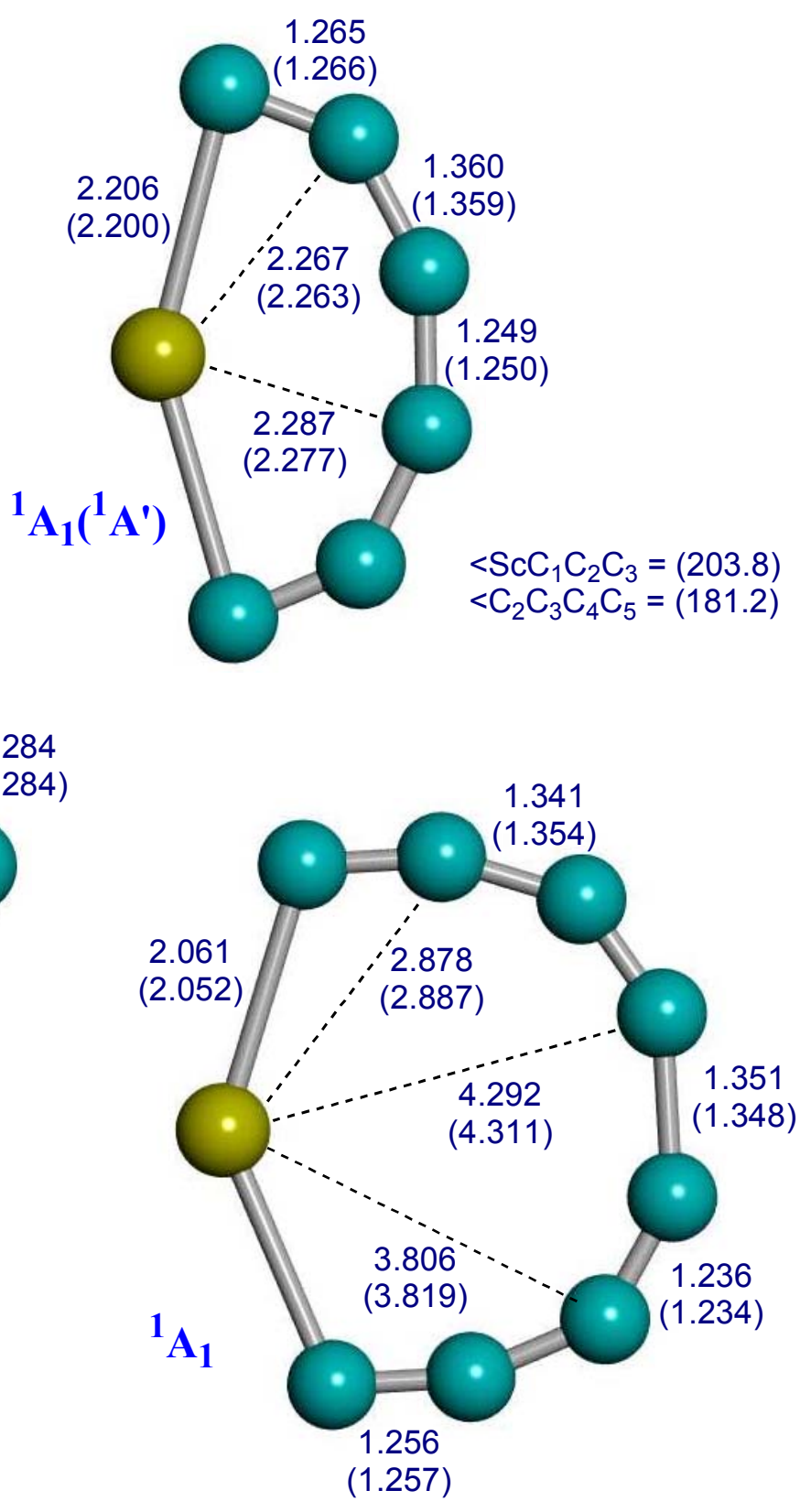

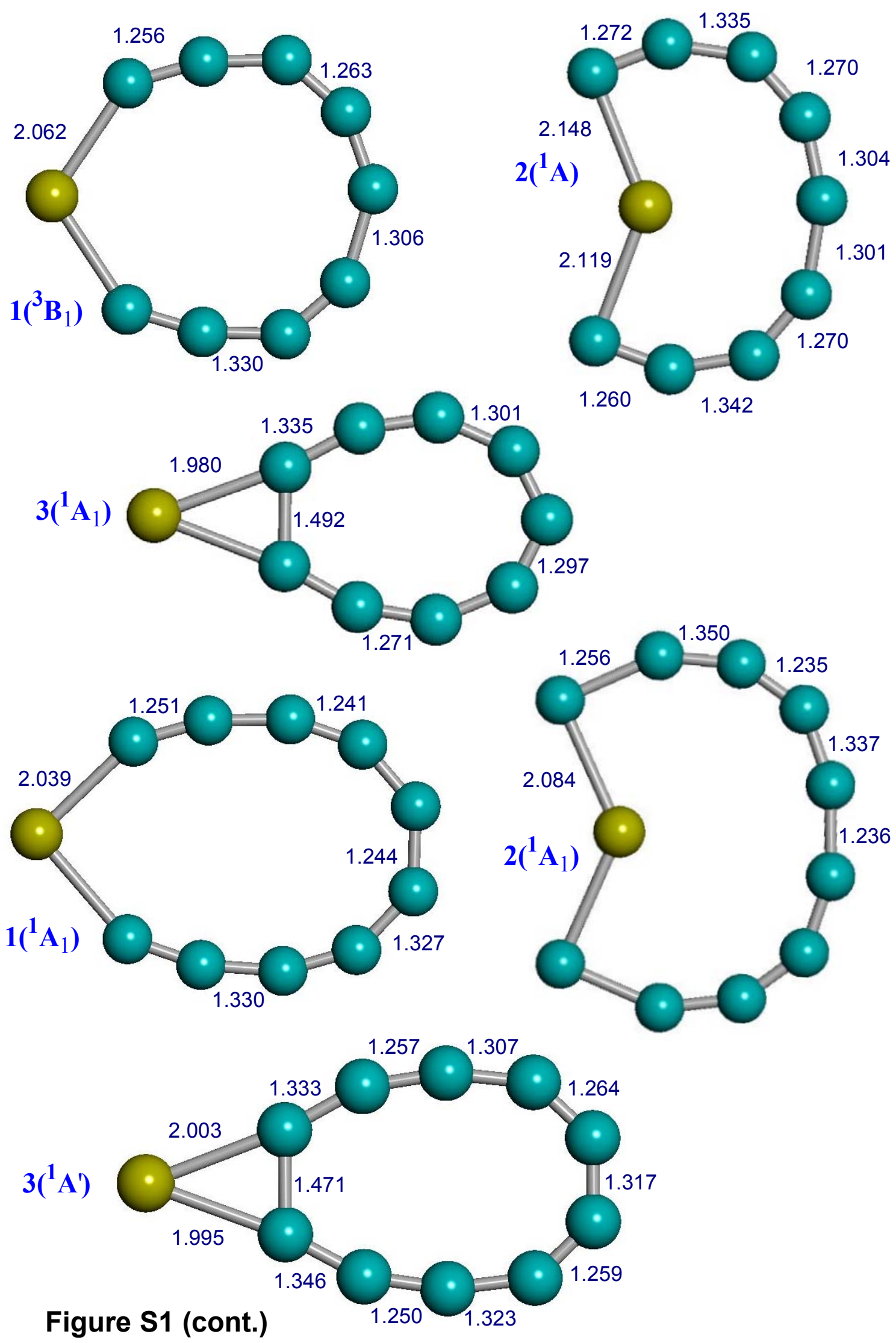

Figure S1. Equilibrium geometries of $\mathrm{ScC}_{n}{ }^{+}$cyclic clusters at the B3LYP/6-311+G(d) and B3LYP/LAN-6+(d) (in parentheses) levels of theory. Distances are given in angstroms and angles in degrees. 

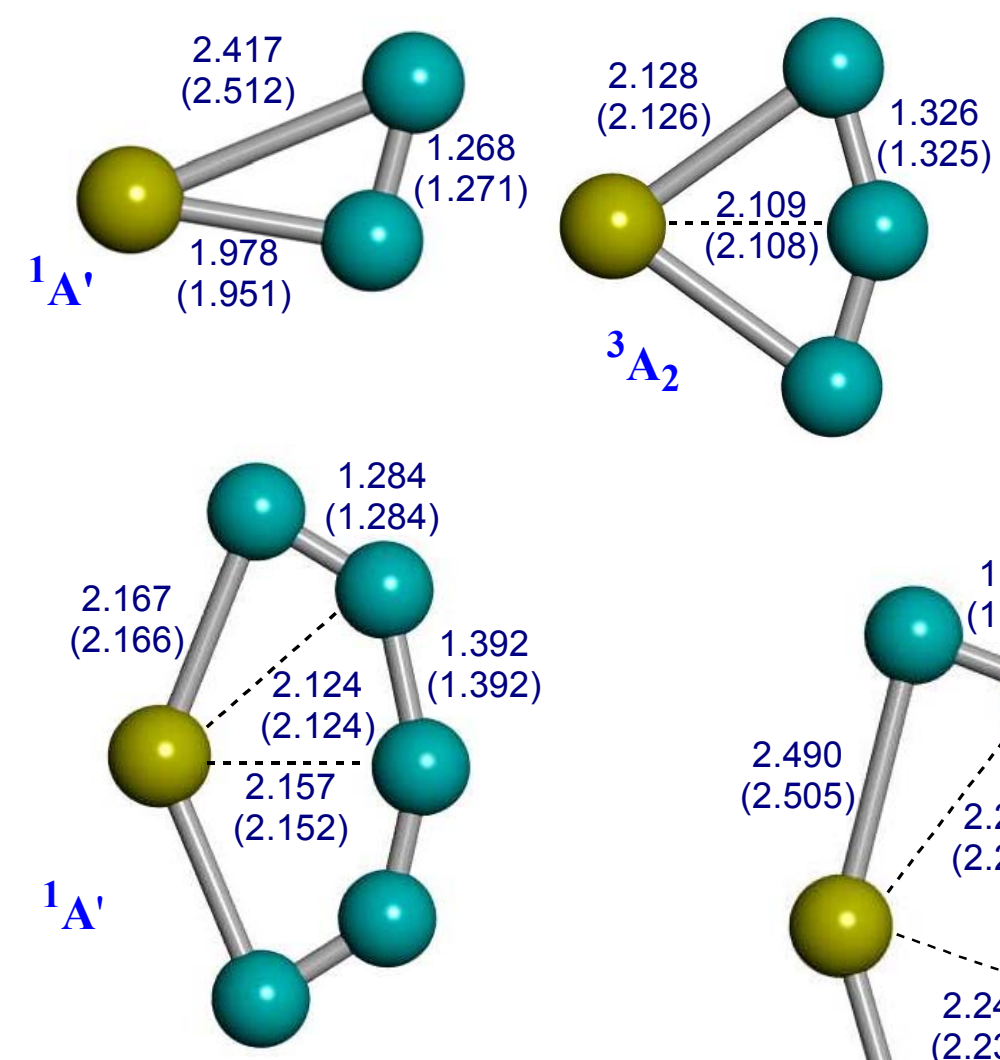

$<\mathrm{ScC}_{1} \mathrm{C}_{2} \mathrm{C}_{3}=239.3$ (239.8)

$<\mathrm{C}_{2} \mathrm{C}_{3} \mathrm{C}_{4} \mathrm{C}_{5}=218.1$ (218.7)

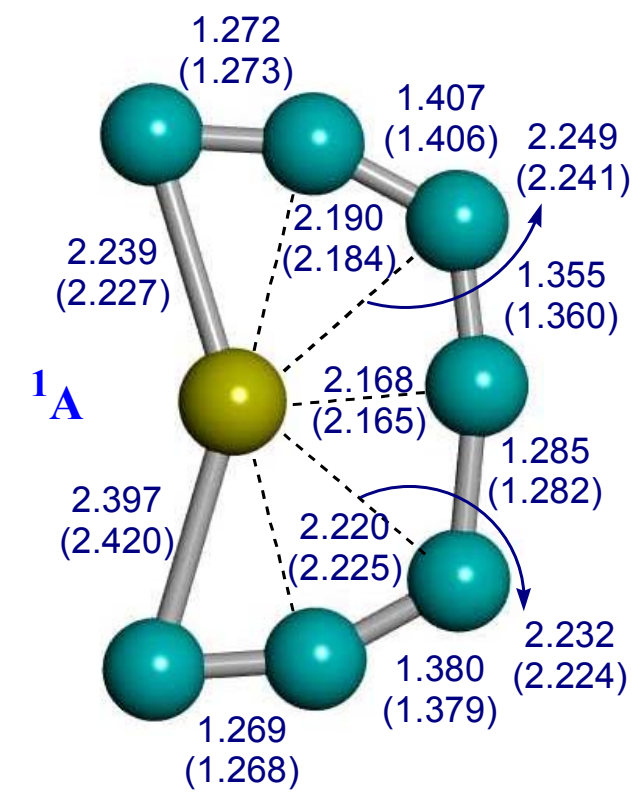

$<\mathrm{C}_{1} \mathrm{C}_{3} \mathrm{C}_{5} \mathrm{C}_{7}=-62.3(-63.5)$

$<\mathrm{C}_{2} \mathrm{C}_{4} \mathrm{C}_{6} \mathrm{C}_{7}=-24.1(-20.3)$

$<\mathrm{C}_{3} \mathrm{C}_{5} \mathrm{C}_{7} \mathrm{C}_{6}=58.3$ (60.8)

Figure S2
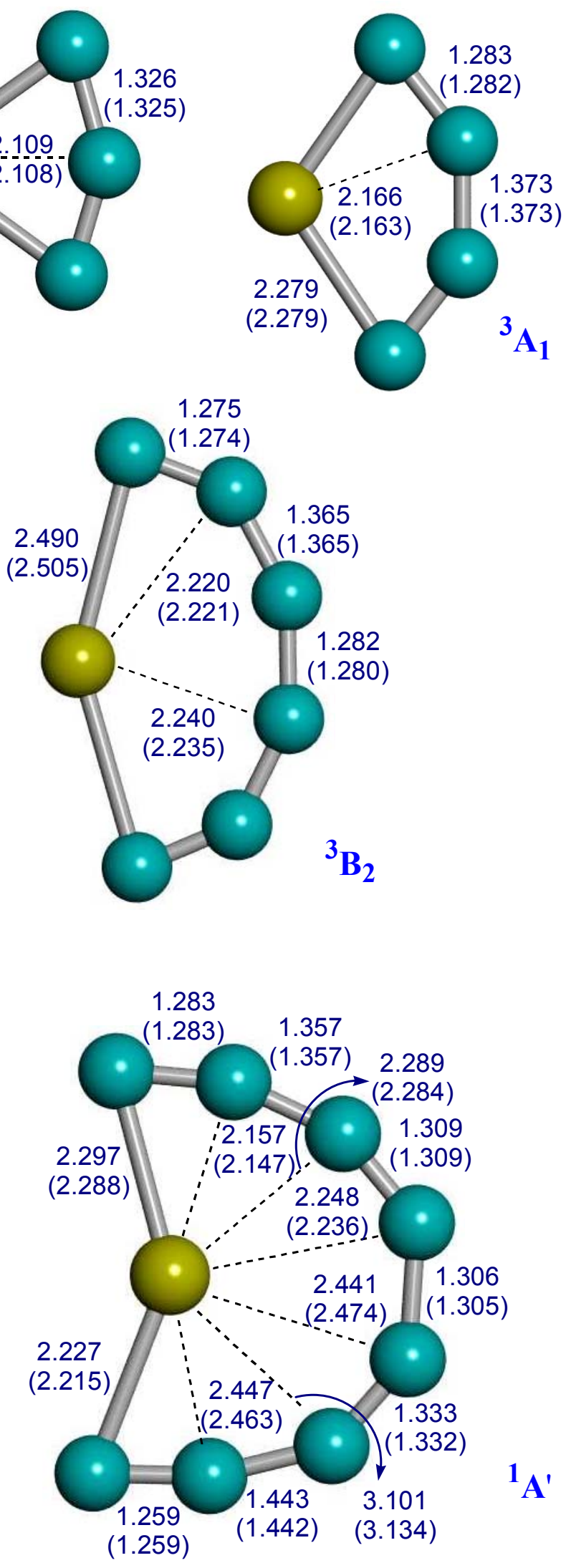

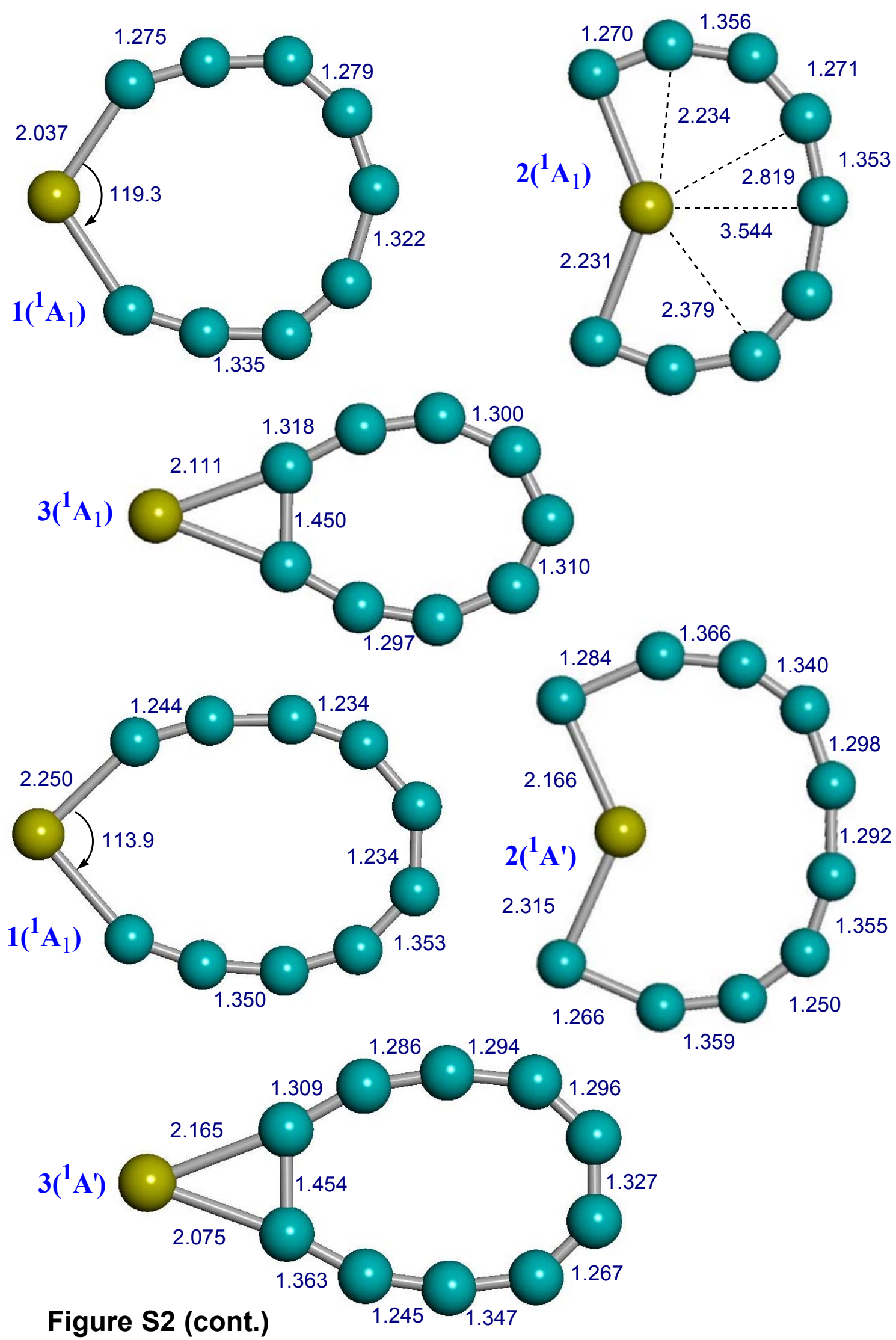

Figure S2. Equilibrium geometries of $\mathrm{ScC}_{\mathrm{n}}{ }^{-}$cyclic clusters at the $\mathrm{B} 3 \mathrm{LYP} / 6-311+\mathrm{G}(\mathrm{d})$ and B3LYP/LAN-6+(d) (in parentheses) levels of theory. Distances are given in angstroms and angles in degrees. 